\title{
The Poisson Algebra of the Invariant Charges of the Nambu-Goto Theory: Casimir Elements
}

\author{
K. Pohlmeyer $\star \star \star ~$
}

Department of Physics and Lawrence Berkeley Laboratory, University of California, Berkeley, CA 94720, USA

\begin{abstract}
The reparametrization invariant "non-local" conserved charges of the Nambu-Goto theory form an algebra under Poisson bracket operation. The center of the formal closure of this algebra is determined. The relation of the central elements to the constraints of the Nambu-Goto theory is clarified.
\end{abstract}

\section{Introduction}

By now it is well known that the classical Nambu-Goto theory of bosonic closed strings moving in $d \geqq 3$ space-time dimensions possesses infinitely many independent, reparametrization invariant, "non-local" conserved charges $\mathscr{Z}_{\mu_{1} \ldots \mu_{N}}^{ \pm(K)}$ which serve as infinitesimal generators of active symmetry transformations $[1,2]$ and which can be carried over to the quantum theory at least in the WKBapproximation [3]. The Poisson algebra $\mathfrak{h}_{\mathscr{P}}^{ \pm}$(for given numerical values of the linear momenta $\mathscr{P}_{\mu}$ ) of these "invariant" charges $\mathscr{Z}_{\mu_{1} \ldots \mu_{N}}^{ \pm(K)}$ has been analyzed in some detail [4]. A maximal abelian subalgebra has been identified for the case $\mathscr{P}^{2}=\mathrm{m}^{2}>0$ and it has been established that there are no Casimir elements in the algebra $\mathfrak{h}_{\mathscr{P}}^{ \pm}$itself [5].

In the present paper I shall determine the Casimir elements contained in the formal closure $\overline{\mathfrak{h} \frac{ \pm}{\mathscr{P}}}$ of $\mathfrak{h} \frac{ \pm}{\mathscr{P}}$ for $\mathscr{P}^{2}=\mathfrak{m}^{2}>0$ and point out their relation to the constraints of the Nambu-Goto theory. As it turns out, there are exactly two such independent Casimir elements: one in $\overline{\mathfrak{h}_{\mathscr{P}}^{+}}$and one in $\overline{\mathfrak{h}_{\mathscr{P}}}$. The corresponding formal series in the Fourier components $A_{\mu}^{ \pm m}(\tau)$ of the string variables $u_{\mu}^{ \pm}(\tau, \sigma)$ $=p_{\mu}(\tau, \sigma) \pm M^{2} x_{\mu}^{\prime}(\tau, \sigma), 0 \leqq \sigma \leqq 2 \pi, u_{\mu}^{ \pm}(\tau, \sigma+2 \pi)=u_{\mu}^{ \pm}(\tau, \sigma), \mu=0,1, \ldots, d-1$, where $\tau$ is a time-like and $\sigma$ is a space-like parameter, can be derived from the integrals

$$
\oint_{\tau=\text { const }} d \sigma\left[\left(u^{+\mu}(\tau, \sigma) u_{\mu}^{+}(\tau, \sigma)\right)^{2}\right]^{1 / 4} \quad \text { and } \quad \oint_{\tau=\text { const }} d \sigma\left[\left(u^{-\mu}(\tau, \sigma) u_{\mu}^{-}(\tau, \sigma)\right)^{2}\right]^{1 / 4},
$$

\footnotetext{
$\star$ On leave of absence from Physics Faculty, University of Freiburg, Federal Republic of Germany $\star \star$ This work was supported by Stiftung Volkswagenwerk, Deutsche Forschungsgemeinschaft and by the Director, Office of Energy Research, Office of High Energy and Nuclear Physics, Division of High Energy Physics of the U.S. Department of Energy under Contract De-AC0376SF0098
} 
respectively. The possibility that there exist additional independent Casimir elements in the algebra of fractions of $\overline{\mathfrak{h}_{\mathscr{P}}^{ \pm}}[6]$ has not completely been ruled out, yet.

Without loss of generality, I shall carry out the proof in the momentum rest frame. For the Fourier decomposition with respect to the variable $\sigma$ this means

$$
u_{\mu}^{ \pm}(\tau, \sigma)=\left(\frac{m}{2 \pi}\right) \delta_{\mu, 0}+\sum_{n}^{\prime} A_{\mu}^{ \pm m}(\tau) e^{i m \sigma}, \quad \mu=0,1, \ldots, d-1 .
$$

The Fourier coefficients $A_{\mu}^{ \pm m}(\tau)$ satisfy the commutation relations

$$
\begin{gathered}
\left\{A_{\mu}^{ \pm m}(\tau), A_{\nu}^{ \pm n}(\tau)\right\}=\mp\left(g_{\mu v} / \pi\right) i m \delta_{0, m+n}, \quad m, n= \pm 1, \pm 2, \ldots, \\
\left\{A_{\mu}^{+m}(\tau), A_{v}^{-n}(\tau)\right\}=0, \quad \mu, v=0,1, \ldots, d-1 .
\end{gathered}
$$

Here the symbol $\sum_{n}^{\prime}$ denotes summation over all integer values of $n$ from $-\infty$ to $+\infty$ with the exception of zero, the symbol $\{$,$\} stands for Poisson bracket$ operation, $g_{\mu \nu}=\operatorname{diag}(+,-, \ldots,-)$, and the string tension parameter $M^{2}$ has been set equal to 1 . I "renormalize" the Fourier coefficients, suppress the $\tau$-dependence and restrict myself to the + combinations:

$$
\begin{gathered}
\alpha_{\mu}^{m}=A_{\mu}^{+m}(\tau) /\left(\frac{m}{2 \pi}\right), \quad m= \pm 1, \pm 2, \ldots, \\
\left\{\alpha_{\mu}^{m}, \alpha_{\nu}^{n}\right\}=-\kappa g_{\mu \nu} i m \delta_{0, m+n}, \quad m, n= \pm 1, \pm 2, \ldots, \quad \kappa \doteqdot \frac{4 \pi}{m^{2}} .
\end{gathered}
$$

Rotational invariance in the momentum rest frame, i.e. vanishing Poisson bracket with the charges $\mathscr{Z}_{0 i j}^{(2)}$ forces any candidate $\mathscr{K}$ for a Casimir element in $\overline{\mathfrak{h}}_{(\mathrm{m}, 0, \ldots, 0)}$ to have the general form

$$
\begin{aligned}
& \mathscr{K}=\mathscr{K}^{(1)}+\mathscr{K}^{(2)}: \\
& \mathscr{K}^{(1)}=\sum_{\substack{a=0 \\
a+r>0}}^{\infty} \sum_{\substack{r=0 \\
k_{1}}}^{\infty} \sum_{k_{1}}^{\prime} \sum_{\ell_{1}}^{\prime} \ldots \sum_{k_{a}}^{\prime} \sum_{\ell_{a}}^{\prime} \sum_{m_{1}}^{\prime} \ldots \sum_{m_{r}}^{\prime}\left(\alpha_{i_{1}}^{k_{1}} \alpha_{i_{1}}^{\ell_{1}}\right) \ldots\left(\alpha_{i_{a}}^{k_{a}} \alpha_{i_{a}}^{\ell_{a}}\right) \alpha_{0}^{m_{1}} \ldots \\
& \ldots \alpha_{0}^{m_{r}} B_{\left(\left(k_{1} \ell_{1}\right) \ldots\left(k_{a} \ell_{a}\right)\right)}^{\left(m_{1} \ldots m_{r}\right)}, \\
& \mathscr{K}^{(2)}= \sum_{a=0}^{\infty} \sum_{r=0}^{\infty} \sum_{n_{1}}^{\prime} \ldots \sum_{n_{d-1}}^{\prime} \sum_{k_{1}}^{\prime} \sum_{\ell_{1}}^{\prime} \ldots \sum_{k_{a}}^{\prime} \sum_{\ell_{a}}^{\prime} \sum_{m_{1}}^{\prime} \ldots \sum_{m_{r}}^{\prime} \alpha_{1}^{n_{1}} \ldots \alpha_{d-1}^{n_{d}-1} \\
& \times\left(\alpha_{i_{1}}^{k_{1}} \alpha_{i_{1}}^{\ell_{1}}\right) \ldots\left(\alpha_{i_{a}}^{k_{a}} \alpha_{i_{a}}^{\ell} a\right) \alpha_{0}^{m_{1}} \ldots \alpha_{0}^{m_{r}} B_{\left.\left[n_{1} \ldots n_{d}-1\right]\left(k_{1} \ell_{1}\right) \ldots\left(k_{a} \ell_{a}\right)\right)}^{\left(m_{1} \ldots m_{r}\right)} .
\end{aligned}
$$

Here summation over the space-like tensor indices $i_{b}, b=1, \ldots, a$ from 1 to $(d-1)$ is understood. The summations over $a$ and $r$ cannot both terminate [5]. The coefficients $B_{\left(\left(k_{1} \ell_{1}\right) \ldots\left(k_{a} \ell_{a}\right)\right)}^{\left(m_{1} \ldots m_{r}\right)}$ and $B_{\left.\left[n_{1} \ldots n_{d}-1\right]\left(k_{1} \ell_{1}\right) \ldots\left(k_{a} \ell a\right)\right)}^{\left(m_{1} \ldots m_{2}\right)}$ are totally symmetric in the upper indices $m_{s}, s=1, \ldots, r$. The latter type of coefficients is totally antisymmetric in the indices $n_{1}, \ldots, n_{d-1}$. Both types of coefficients are symmetric under the interchange of $k_{b}$ and $\ell_{b}, b=1, \ldots, a$ and under the interchange of any two pairs $\left(k_{b}, \ell_{b}\right)$ and $\left(k_{c}, \ell_{c}\right), b, c=1, \ldots, a$. They transform under permutations of the symmetric group $\mathfrak{\subseteq}^{2 a} / \mathfrak{S}^{2 a+d-1}$ according to a (in general reducible) representation corresponding to partitions of $2 a / 2 a+d-1$ into no more than/exactly $(d-1)$ even/odd integers. The corresponding standard tableaux contain in every row along with an index $k_{b}$ also its partner index $\ell_{b}$.

On account of the different content of Fourier coefficients carrying space-like indices, each one of the two formal series $\mathscr{K}^{(1)}$ and $\mathscr{K}^{(2)}$ separately must have vanishing Poisson brackets with the generators of infinitesimal reparametrizations 
and with all invariant charges $\mathscr{Z}_{\mu_{1} \ldots \mu_{N}}^{(K)}$. Consequently, I shall treat the two types of candidates for the Casimir elements separately starting with the (spatially) pseudoscalar type $\mathscr{K}^{(2)}$.

\section{Pseudoscalar Type}

In this section I shall show that there are no Casimir elements of the pseudoscalar type,

$$
\begin{aligned}
\mathscr{K}^{(2)}= & \sum_{a=0}^{\infty} \sum_{r=0}^{\infty} \sum_{n_{1}}^{\prime} \ldots \sum_{n_{d-1}}^{\prime} \sum_{k_{1}}^{\prime} \sum_{\ell_{1}}^{\prime} \ldots \sum_{k_{a}}^{\prime} \sum_{\ell_{a}}^{\prime} \sum_{m_{1}}^{\prime} \ldots \sum_{m_{r}}^{\prime} \alpha_{1}^{n_{1}} \ldots \alpha_{d-1}^{n_{d}-1}\left(\alpha_{i_{1}}^{k_{1}} \alpha_{i_{1}}^{\ell_{1}}\right) \ldots \\
& \ldots\left(\alpha_{i_{a}}^{k_{a}} \alpha_{i_{a}}^{\ell_{a}}\right) \alpha_{0}^{m_{1}} \ldots \alpha_{0}^{m_{r}} B_{\left.\left[n_{1} \ldots n_{d}-1\right]\left(m_{1} m_{1}\right) \ldots\left(k_{a} \ell_{a}\right)\right) .}^{\left(m_{1}\right)}
\end{aligned}
$$

Reparametrization invariance implies that the coefficients $B_{\left[n_{1} \ldots n_{d}-1\right]\left(\left(k_{1} \ell_{1}\right) \ldots\left(k_{a} \ell_{a}\right)\right)}^{\left(m_{1} \ldots m_{r}\right)}$ satisfy the following equations

i)

$$
\text { i) } \quad \begin{aligned}
( & \left.n_{1}+\ldots+n_{d-1}+k_{1}+\ell_{1}+\ldots+k_{a}+\ell_{a}+m_{1}+\ldots+m_{r}\right) \\
& \times B_{\left[n_{1} \ldots n_{d}-1\right]\left(\left(k_{1} \ell_{1}\right) \ldots\left(k_{a} \ell_{a}\right)\right)}^{\left(m_{1} \ldots m_{r}\right)}=0, \\
(r+1) & m_{r+1} B_{\left.\left[n_{1} \ldots n_{d}-1\right]\left(k_{1} \ell_{1}\right) \ldots\left(k_{a} \ell_{a}\right)\right)}^{\left(m_{1} \ldots m_{r}\right)} \\
& +\sum_{s=1}^{r}\left(m_{s}+m_{r+1}\right) B_{\left.\left[n_{1} \ldots n_{d}-1\right]\left(k_{1} \ell_{1}\right) \ldots\left(k_{a} \ell_{a}\right)\right)}^{\left(m_{1} \ldots\left\{m_{s}+m_{r}+m_{r}\right)\right.} \\
& +\sum_{j=1}^{d-1}\left(n_{j}+m_{r+1}\right) B_{\left[n_{1} \ldots\left\{n_{j}+m_{r+1}\right\} \ldots n_{d-1}\right]\left(\left(k_{1} \ell_{1}\right) \ldots\left(k_{a} \ell a\right)\right)}^{\left(m_{1} \ldots m_{r}\right)} \\
& +\sum_{b=1}^{a}\left\{\left(k_{b}+m_{r+1}\right) B_{\left[n_{1} \ldots n_{d}-1\right]\left(\left(k_{1} \ell_{1}\right) \ldots\left(\left\{k_{b}+m_{r+1}\right\} \ell_{b}\right) \ldots\left(k_{a} \ell a\right)\right)}^{\left(m_{1} \ldots m_{r}\right)}\right. \\
& \left.+\left(\ell_{b}+m_{r+1}\right) B_{\left[n_{1} \ldots n_{d}-1\right]\left(\left(k_{1} \ell_{1}\right) \ldots\left(k_{b}\left\{\ell_{b}+m_{r+1}\right\}\right) \ldots\left(k_{a} \ell_{a}\right)\right)}^{\left(m_{1} \ldots m_{r}\right)}\right\}=0
\end{aligned}
$$

with the obvious interpretation of the left-hand sides for the cases $r=0$ and/or $a=0$.

The former equations are solved by

$$
\begin{aligned}
B_{\left[n_{1} \ldots n_{d}-1\right]\left(\left(k_{1} \ell_{1}\right) \ldots\left(k_{a} \ell_{a}\right)\right)=}^{\left(m_{1} \ldots m_{r}\right)} & \delta_{0,\left(n_{1}+\ldots+n_{d-1}+k_{1}+\ell_{1}+\ldots+k_{a}+\ell_{a}+m_{1}+\ldots+m_{r}\right)} \\
& \times \hat{B}_{\left[n_{1} \ldots n_{d}-1\right]\left(\left(k_{1} \ell_{1}\right) \ldots\left(k_{a} \ell_{a}\right)\right)}^{\left(m_{1} \ldots m_{2}\right)} .
\end{aligned}
$$

For a given non-negative integer value of $a$, the latter equations enforce the vanishing of all coefficients

once the coefficients

$$
\begin{gathered}
B_{\left[n_{1} \ldots n_{d}-1\right]\left(\left(k_{1} \ell_{1}\right) \ldots\left(k_{a} \ell_{a}\right)\right)}^{\left(m_{1} \ldots m_{r}\right)} k_{b}, \ell_{c}, m_{s}, n_{j}= \pm 1, \pm 2, \ldots, \quad b, c=1, \ldots, a, \\
s=1, \ldots, r, \quad j=1, \ldots, d-1,
\end{gathered}
$$

vanish.

$$
\begin{gathered}
B_{\left[n_{1} \ldots n_{d-1}\right]\left(\left(k_{1} \ell_{1}\right) \ldots\left(k_{a} \ell a\right)\right)} k_{b}, \ell_{c}, n_{j}= \pm 1, \pm 2, \ldots, \\
b, c=1, \ldots, a, \quad j=1, \ldots, d-1
\end{gathered}
$$

Therefore the claim will be proved if I can show that the assumption of the existence of a minimal non-negative finite integer $A$ with

$$
\sum_{n_{1}}^{\prime} \ldots \sum_{n_{d}-1}^{\prime} \sum_{k_{1}}^{\prime} \sum_{\ell_{1}}^{\prime} \ldots \sum_{k_{A}}^{\prime} \sum_{\ell_{A}}^{\prime} \alpha_{1}^{n_{1}} \ldots \alpha_{d-1}^{n_{d}-1}\left(\alpha_{i_{1}}^{k_{1}} \alpha_{i_{1}}^{\ell_{1}}\right) \ldots\left(\alpha_{i_{A}}^{k_{A}} \alpha_{i_{A}{ }_{A}}^{A_{1}}\right) B_{\left[n_{1} \ldots n_{d-1}\right]\left(\left(k_{1} \ell_{1}\right) \ldots\left(k_{A} \ell A\right)\right)} \neq 0
$$

leads to a contradiction. 
By its defining property $\mathscr{K}^{(2)}$ has vanishing Poisson brackets with any element of $\mathfrak{h}_{(\mathfrak{m}, 0, \ldots, 0)}$, for instance with the "renormalized" invariant charges $\underbrace{}_{\underbrace{(2)}_{i} \mathscr{Z}^{(2)} j}$,
$i, j \in\{1, \ldots, d-1\}$ :

$$
\mathscr{Z}_{i=\ldots}^{(2) \ldots 0 j}=-(2 \pi)^{2} \sum_{n}^{\prime} \frac{\alpha_{i}^{-n} \alpha_{j}^{n}}{(i n)^{p}}+O_{p}\left(\alpha_{i}^{k} \alpha_{j}^{\ell}, \alpha_{0}^{m}\right) .
$$

Here $O_{p}\left(\alpha_{i}^{k} \alpha_{j}^{\ell}, \alpha_{0}^{m}\right)$ denotes the contribution of first degree in the variables $\alpha_{i}^{k} \alpha_{j}^{\ell}$, $k, \ell= \pm 1, \pm 2, \ldots$ and of first and higher degree in the Fourier coefficients $\alpha_{0}^{m}$, $m= \pm 1, \pm 2, \ldots$. As a consequence of i) the vanishing Poisson bracket between $\mathscr{K}^{(2)}$ and $\underset{(2 p) \times}{\mathscr{Z}_{j}^{(2)} \ldots 0 j}$ (no summation over $j$ !) and of ii) the minimality requirement imposed on the number $A$, I obtain the following equation:

$$
\begin{aligned}
& \sum_{n_{1}}^{\prime} \ldots \sum_{n_{d-1}}^{\prime} \sum_{k_{1}}^{\prime} \sum_{\ell_{1}}^{\prime} \ldots \sum_{k_{A}}^{\prime} \sum_{\ell_{A}}^{\prime} \alpha_{1}^{n_{1}} \ldots \alpha_{d-1}^{n_{d}-1}\left(\alpha_{i_{1}}^{k_{1}} \alpha_{i_{1}}^{\ell_{1}}\right) \ldots\left(\alpha_{i_{A}}^{k_{A}} \alpha_{i_{A} \ell_{A}}\right) \\
& \quad \times B_{\left[n_{1} \ldots n_{d-1}\right]\left(\left(k_{1} \ell_{1}\right) \ldots\left(k_{A} \ell_{A}\right)\right)}\left\{\frac{1}{n_{j}^{2 p-1}}+A\left(\frac{1}{k_{A}^{2 p-1}}+\frac{1}{\ell_{A}^{2 p-1}}\right) \delta_{i_{A}, j}\right\}=0 .
\end{aligned}
$$

This equation implies for arbitrary positive integer values of $p$,

$$
\begin{aligned}
& \frac{1}{n_{j}^{2 p-1}} \cdot \sum_{n_{1}}^{\prime} \ldots \check{\Sigma}_{n_{j}}^{\prime} \ldots \sum_{n_{d-1}}^{\prime} \sum_{k_{1}}^{\prime} \sum_{\ell_{1}}^{\prime} \ldots \sum_{k_{A}}^{\prime} \sum_{\ell_{A}}^{\prime} \alpha_{1}^{n_{1}} \ldots \check{\alpha}_{j}^{n_{j}} \ldots \alpha_{d-1}^{n_{d}-1} \\
& \times\left(\alpha_{i_{1} \neq j}^{k_{1}} \alpha_{i_{1} \neq j}^{\ell_{1}}\right) \ldots\left(\alpha_{i_{A} \neq j}^{k_{A}} \alpha_{i_{A} \neq j}^{\ell_{A}}\right) B_{\left[n_{1} \ldots n_{d-1}\right]\left(\left(k_{1} \ell_{1}\right) \ldots\left(k_{A} \ell_{A}\right)\right)}=0, \\
& \left(\frac{1}{n_{j}^{2 p-1}}+\frac{1}{k_{A}^{2 p-1}}+\frac{1}{\ell_{A}^{2 p-1}}\right) \\
& \times \sum_{n_{1}}^{\prime} \ldots \check{\sum}_{n_{j}}^{\prime} \ldots \sum_{n_{d-1}}^{\prime} \sum_{k_{1}}^{\prime} \sum_{\ell_{1}}^{\prime} \ldots \sum_{k_{A-1}}^{\prime} \sum_{\ell_{A-1}}^{\prime} \alpha_{1}^{n_{1}} \ldots \check{\alpha}_{j}^{n_{j}} \ldots \\
& \ldots \alpha_{d-1}^{n_{d}-1}\left(\alpha_{i_{1} \neq j}^{k_{1}} \alpha_{i_{1} \neq j}^{\ell_{1}}\right) \ldots\left(\alpha_{i_{A}-1 \neq j}^{k_{A-1}} \alpha_{i_{A-1} \neq j}^{\ell_{A-1}}\right) \\
& \times\left(B_{\left.\left[n_{1} \ldots n_{d-1}\right]\left(k_{1} \ell_{1}\right) \ldots\left(k_{A} \ell_{A}\right)\right)}\right)_{\left(n_{j} k_{A} \ell_{A}\right)}=0, \\
& \left(\frac{1}{n_{j}^{2 p-1}}+\frac{1}{k_{A-1}^{2 p-1}}+\frac{1}{\ell_{A-1}^{2 p-1}}+\frac{1}{k_{A}^{2 p-1}}+\frac{1}{\ell_{A}^{2 p-1}}\right) \\
& \times \sum_{n_{1}}^{\prime} \ldots \check{\sum}_{n_{j}}^{\prime} \ldots \sum_{n_{d-1}}^{\prime} \sum_{k_{1}}^{\prime} \sum_{\ell_{1}}^{\prime} \ldots \sum_{k_{A-2}}^{\prime} \sum_{\ell_{A-2}^{\prime}}^{\prime} \alpha_{1}^{n_{1}} \ldots \check{\alpha}_{j}^{n_{j}} \ldots \\
& \ldots \alpha_{d-1}^{n_{d-1}}\left(\alpha_{i_{1} \neq j}^{k_{1}} \alpha_{i_{1} \neq j}^{\ell_{1}}\right) \ldots\left(\alpha_{i_{A-2} \neq j}^{k_{A}-2} \alpha_{i_{A-2}}^{\ell_{A-2}}\right) \\
& \times\left(B_{\left[n_{1} \ldots n_{d}-1\right]\left(\left(k_{1} \ell_{1}\right) \ldots\left(k_{A} \ell_{A}\right)\right)}\right)_{\left(n_{j} k_{A}-1 \ldots \ell_{A}\right)}=0, \\
& \left(\frac{1}{n_{j}^{2 p-1}}+\frac{1}{k_{1}^{2 p-1}}+\frac{1}{\ell_{1}^{2 p-1}}+\ldots+\frac{1}{k_{A}^{2 p-1}}+\frac{1}{\ell_{A}^{2 p-1}}\right) \sum_{n_{1}}^{\prime} \ldots \check{\check{L}}_{n_{J}}^{\prime} \ldots \sum_{n_{d-1}^{\prime}}^{\prime} \alpha_{1}^{n_{1}} \ldots \check{\alpha}_{j}^{n_{J}} \ldots \\
& \ldots \alpha_{d-1}^{n_{d}-1}\left(B_{\left[n_{1} \ldots n_{d-1}\right]\left(\left(k_{1} \ell_{1}\right) \ldots\left(k_{A} \ell_{A}\right)\right)}\right)_{\left(n_{j} k_{1} \ell_{1} \ldots k_{A} \ell_{A}\right)}=0 \text {. }
\end{aligned}
$$


Here the symbol $\check{a}$ indicates omission of the object or operation $a$. The notation $\left(\alpha_{i_{b} \neq j}^{k_{b}} \alpha_{i_{b} \neq j}^{\ell_{b}}\right)$ is to be understood as meaning

$$
\sum_{j \neq i_{b}=1}^{d-1} \alpha_{i_{b}}^{k_{b}} \alpha_{i_{b}}^{\ell_{b}}
$$

The symbol $(B \ldots)_{\left(n_{j} k_{b} \ell_{b} \ldots k_{a} \ell_{a}\right)}$ stands for symmetrization of $B \ldots$ with respect to the indices $n_{j}, k_{b}, \ell_{b}, \ldots, k_{a}, \ell_{a}$. Since for fixed non-negative integers $t$ the simultaneous equations

$$
x_{1}^{2 p-1}+x_{2}^{2 p-1}+\ldots+x_{2 t+1}^{2 p-1}=0, \quad p=1,2,3, \ldots
$$

do not have solutions with all $x_{i}, i=1, \ldots, 2 t+1$ different from zero, I conclude

$$
\begin{aligned}
& \sum_{n_{1}}^{\prime} \ldots \sum_{n_{J}}^{\prime} \ldots \sum_{n_{d}-1}^{\prime} \sum_{k_{1}}^{\prime} \sum_{\ell_{1}}^{\prime} \ldots \sum_{k_{A}}^{\prime} \sum_{\ell_{A}}^{\prime} \alpha_{1}^{n_{1}} \ldots \alpha_{j}^{n_{j}} \ldots \alpha_{d-1}^{n_{d}-1}\left(\alpha_{i_{1} \neq j}^{k_{1}} \alpha_{i_{1} \neq j}^{\ell_{1}}\right) \ldots\left(\alpha_{i_{A} \neq j}^{k_{A}} \alpha_{i_{A} \neq j}^{\ell_{A}}\right) \\
& \times B_{\left[n_{1} \ldots n_{d-1}\right]\left(\left(k_{1} \ell_{1}\right) \ldots\left(k_{A} \ell_{A}\right)\right)}=0 \text {, } \\
& \sum_{n_{1}}^{\prime} \ldots \sum_{n_{J}}^{\prime} \ldots \sum_{n_{d-1}}^{\prime} \sum_{k_{1}}^{\prime} \sum_{\ell_{1}}^{\prime} \ldots \sum_{k_{A}}^{\prime} \sum_{\ell_{A}}^{\prime} \alpha_{1}^{n_{1}} \ldots \alpha_{j}^{n_{J}} \ldots \alpha_{d-1}^{n_{d}-1}\left(\alpha_{i_{1} \neq j}^{k_{1}} \alpha_{i_{1} \neq j}^{\ell_{1}}\right) \ldots \\
& \ldots\left(\alpha_{i_{A-1} \neq j}^{k_{A-1}} \alpha_{i_{A-1}^{\ell} \neq j}^{\ell_{A-1}}\right)\left(\alpha_{j}^{k_{A}} \alpha_{j}^{\ell A}\right) B_{\left[n_{1} \ldots n_{d-1}\right]\left(\left(k_{1} \ell_{1}\right) \ldots\left(k_{A} \ell_{A}\right)\right)}=0, \\
& \sum_{n_{1}}^{\prime} \ldots \sum_{n_{j}}^{\prime} \ldots \sum_{n_{d-1}}^{\prime} \sum_{k_{1}}^{\prime} \sum_{\ell_{1}}^{\prime} \ldots \sum_{k_{A}}^{\prime} \sum_{\ell_{A}}^{\prime} \alpha_{1}^{n_{1}} \ldots \alpha_{j}^{n_{j}} \ldots \alpha_{d-1}^{n_{d-1}}\left(\alpha_{j}^{k_{1}} \alpha_{j}^{\ell_{1}}\right) \ldots\left(\alpha_{j}^{k_{A}} \alpha_{j}^{\ell_{A} A}\right) \\
& \times B_{\left[n_{1} \ldots n_{d-1}\right]\left(\left(k_{1} \ell_{1}\right) \ldots\left(k_{A} \ell_{A}\right)\right)}=0,
\end{aligned}
$$

and finally

$$
\begin{aligned}
& \sum_{n_{1}}^{\prime} \ldots \sum_{n_{d-1}}^{\prime} \sum_{k_{1}}^{\prime} \sum_{\ell_{1}}^{\prime} \ldots \sum_{k_{A}}^{\prime} \sum_{\ell_{A}}^{\prime} \alpha_{1}^{n_{1}} \ldots \alpha_{d-1}^{n_{d}-1}\left(\alpha_{i_{1}}^{k_{1}} \alpha_{i_{1}}^{\ell_{1}}\right) \ldots\left(\alpha_{i_{A}}^{k_{A}} \alpha_{i_{A} \ell_{A}}^{\ell_{A}}\right) \\
& \quad \times B_{\left[n_{1} \ldots n_{d}-1\right]\left(\left(k_{1} \ell_{1}\right) \ldots\left(k_{A} \ell_{A}\right)\right)}=0 .
\end{aligned}
$$

However, this is a contradiction to the assumption

$$
\begin{aligned}
& \sum_{n_{1}}^{\prime} \ldots \sum_{n_{d-1}}^{\prime} \sum_{k_{1}}^{\prime} \sum_{\ell_{1}}^{\prime} \ldots \sum_{k_{A}}^{\prime} \sum_{\ell_{A}}^{\prime} \alpha_{1}^{n_{1}} \ldots \alpha_{d-1}^{n_{d}-1}\left(\alpha_{i_{1}}^{k_{1}} \alpha_{i_{1}}^{\ell_{1}}\right) \ldots\left(\alpha_{i_{A}}^{k_{A}} \alpha_{i_{A} \ell_{A}}^{\ell_{A}}\right) \\
& \quad \times B_{\left[n_{1} \ldots n_{d-1}\right]\left(\left(k_{1} \ell_{1}\right) \ldots\left(k_{A} \ell_{A}\right)\right)} \neq 0 .
\end{aligned}
$$

Thus the claim is proved: there are no Casimir elements of the pseudoscalar type.

\section{Scalar Type}

In this section I shall show that any Casimir element of the scalar type contained in the formal closure of $\mathfrak{h}_{(\mathfrak{m}, 0, \ldots, 0)}$ is a function of one special Casimir element $\mathscr{C}$,

$$
\mathscr{C}=\sum_{a=1}^{\infty} \frac{\Gamma\left(-\frac{1}{2}+a\right)}{\Gamma\left(\frac{1}{2}\right) a !} \mathscr{I}_{a}
$$

with the mutually "commuting" limiting elements $\mathscr{I}_{a}$ in $\overline{\mathfrak{h}}_{(\mathfrak{m}, 0, \ldots, 0)}$,

$$
\begin{aligned}
\mathscr{I}_{a}= & \sum_{r=0}^{\infty} \frac{(-1)^{r}}{r !} \frac{\Gamma(2 a-1+r)}{\Gamma(2 a-1)} \sum_{k_{1}}^{\prime} \sum_{\ell_{1}}^{\prime} \ldots \sum_{k_{a}}^{\prime} \sum_{\ell_{a}}^{\prime} \sum_{m_{1}}^{\prime} \ldots \sum_{m_{r}}^{\prime}\left(\alpha_{i_{1}}^{k_{1}} \alpha_{i_{1}}^{\ell_{1}}\right) \ldots\left(\alpha_{i_{a}}^{k_{a}} \alpha_{i_{a}}^{\ell a}\right) \\
& \times \alpha_{0}^{m_{1}} \ldots \alpha_{0}^{m_{r}} \delta_{0,\left(k_{1}+\ell_{1}+\ldots+k_{a}+\ell_{a}+m_{1}+\ldots+m_{r}\right)}, \quad a=1,2, \ldots
\end{aligned}
$$


Here the limiting elements $\mathscr{I}_{a}$ can be expressed in terms of the "renormalized" invariant charges as follows:

$$
\mathscr{I}_{1}=\sum_{k}^{\prime} \frac{1}{(i k)} \frac{1}{2 \pi i} \underset{z}{\oint_{-i k}} d z \mathscr{F}^{(1)}(z)
$$

with

$$
\begin{gathered}
\mathscr{F}^{(1)}(z)=\frac{-1}{(2 \pi)^{2}} \sum_{p=0}^{\infty} z^{p} \mathscr{Z}_{i}^{\mathcal{Y}_{i \times}^{(2)} \ldots 0, i} \\
\mathscr{I}_{2}=\sum_{k} \sum_{\ell}^{\prime} \sum_{m}^{\prime} \frac{1}{(i m)} \frac{1}{2 \pi i} \oint_{\varepsilon / 2} \oint_{\left|z_{1}-i k\right|<\varepsilon} d z_{1} \frac{1}{2 \pi i} \\
\times \oint_{\varepsilon / 8<\left|z_{2}-i \ell\right|<\varepsilon / 4} d z_{2} \frac{1}{2 \pi i} \oint_{0<\left|z_{3}-i m\right|<\varepsilon / 8} d z_{3} \mathscr{F}^{(2)}\left(z_{1}, \cdot, z_{3}\right)
\end{gathered}
$$

with

$$
\mathscr{F}^{(2)}\left(z_{1}, z_{2}, z_{3}\right)=\frac{1}{(2 \pi)^{2}} \sum_{p=0}^{\infty} \sum_{q=0}^{\infty} \sum_{r=0}^{\infty} z_{1}^{p} z_{2}^{q} z_{3}^{r} \mathscr{Z}_{j j}^{(2)} \underbrace{0 \ldots 0}_{q \times} \underbrace{0 \ldots 0}_{p \times} i \underbrace{0 \ldots 0}_{r \times} ;
$$

and so forth.

More specifically, I shall show that for each Casimir element

$$
\begin{aligned}
\mathscr{K}^{(1)}= & \sum_{\substack{a=0 \\
a+r>0}}^{\infty} \sum_{\substack{r=0 \\
k_{1}}}^{\infty} \sum_{k_{1}}^{\prime} \sum_{\ell_{1}}^{\prime} \ldots \sum_{k_{a}}^{\prime} \sum_{\ell_{a}}^{\prime} \sum_{m_{1}}^{\prime} \ldots \sum_{m_{r}}^{\prime}\left(\alpha_{i_{1}}^{k_{1}} \alpha_{i_{1}}^{\ell_{1}}\right) \ldots\left(\alpha_{i_{a}}^{k_{a}} \alpha_{i_{a}}^{\ell_{a}}\right) \\
& \times \alpha_{0}^{m_{1}} \ldots \alpha_{0}^{m_{r}} B_{\left(\left(k_{1} \ell_{1}\right) \ldots\left(k_{a} \ell_{a}\right)\right)}^{\left(m_{1} \ldots m_{r}\right)},
\end{aligned}
$$

one can find numbers $\beta_{a}, a=1,2, \ldots$ such that

$$
\mathscr{K}^{(1)}-\sum_{a=1}^{\infty} \beta_{\alpha} \mathscr{C}^{a}=0 .
$$

Denote the coefficients of the formal series $\mathscr{K}^{(1)}-\sum_{a=1}^{\infty} \beta_{a} \mathscr{C}^{\alpha}$ by $\widetilde{B}_{\left(\left(k_{1} \ell_{1}\right) \ldots\left(m_{r} \ell\right.\right.}^{\left.\left(m_{1}\right)\right)}$ :

$$
\begin{aligned}
& \mathscr{K}^{(1)}-\sum_{a=1}^{\infty} \beta_{a} \mathscr{C}^{a}=\sum_{\substack{a=0 \\
a+r}}^{\infty} \sum_{\substack{r=0 \\
n_{1}}}^{\infty} \sum_{k_{1}}^{\prime} \sum_{\ell_{1}}^{\prime} \ldots \sum_{k_{a}}^{\prime} \sum_{\ell_{a}}^{\prime} \sum_{m_{1}}^{\prime} \ldots \sum_{m_{r}}^{\prime}\left(\alpha_{i_{1}}^{k_{1}} \alpha_{i_{1}}^{\ell_{1}}\right) \ldots \\
& \ldots\left(\alpha_{i_{a}}^{k_{a} a} \alpha_{i_{a}}^{\ell a}\right) \alpha_{0}^{m_{1}} \ldots \alpha_{0}^{m_{r}} \widetilde{B}_{\left(\left(k_{1} \ell_{1}\right) \ldots\left(k_{a} \ell_{a}\right)\right)}^{\left(m_{1} \ldots m_{r}\right)} .
\end{aligned}
$$

The coefficients $\widetilde{B}_{\ldots}^{\ldots}$ have the same symmetry properties as the coefficients $B_{\ldots}^{\ldots}$ appearing in the formal series of $\mathscr{K}^{(1)}$. The numbers $\beta_{a}, a=1,2, \ldots$ are adjusted such that

$$
\widetilde{B}_{((1,-1) \ldots(1,-1))}^{a \times}=0
$$


Invariance under local reparametrizations imposes the following conditions on the coefficients

$$
\begin{aligned}
& \text { i) } \quad\left(k_{1}+\ell_{1}+\ldots+k_{a}+\ell_{a}+m_{1}+\ldots+m_{r}\right) \widetilde{B}_{\left(\left(k_{1} \ell_{1}\right) \ldots\left(k_{a} \ell_{a}\right)\right.}^{\left(m_{1} \ldots m_{r}\right)}=0, \\
& \text { ii) } \quad(1+r) m_{r+1} \widetilde{B}_{\left(\left(k_{1} \ell_{1}\right) \ldots\left(k_{a} \ell a\right)\right)}^{\left(m_{1} \ldots m_{r}+\ell_{1}\right)}+\sum_{s=1}^{r}\left(m_{s}+m_{r+1}\right) \widetilde{B}_{\left(\left(k_{1} \ell_{1}\right) \ldots\left(k_{a} \ell_{a}\right)\right)}^{\left.\left(m_{1} \ldots m_{s}+m_{r+1}\right\} \ldots m_{r}\right)} \\
& +\sum_{b=1}^{a}\left\{\left(k_{b}+m_{r+1}\right) \widetilde{B}_{\left(\left(k_{1} \ell_{1}\right) \ldots\left(\left\{k_{b}+m_{r+1}\right\} \ell_{b}\right) \ldots\left(k_{a} \ell a\right)\right)}^{\left(m_{1} \ldots m_{r}\right)}\right. \\
& \left.+\left(\ell_{b}+m_{r+1}\right) \widetilde{B}_{\left(\left(k_{1} \ell_{1}\right) \ldots\left(m_{b}\right)\right.}^{\left.\left.\left(m_{1} \ldots \ell_{b}+m_{r+1}\right\}\right) \ldots\left(k_{a} \ell_{a}\right)\right)}\right\}=0
\end{aligned}
$$

with the obvious interpretation of the left-hand sides for the cases $r=0$ or $a=0$. The former conditions are solved by

$$
\widetilde{B}_{\left(\left(k_{1} \ell_{1}\right) \ldots\left(k_{a} \ell a\right)\right)}^{\left(m_{1} \ldots m_{r}\right)}=\delta_{0,\left(k_{1}+\ell_{1}+\ldots+k_{a}+\ell_{a}+m_{1}+\ldots+m_{r}\right)} \hat{B}_{\left(\left(k_{1} \ell_{1}\right) \ldots\left(k_{a} \ell a\right)\right)}^{\left(m_{1} \ldots m_{r}\right)}
$$

in particular, because of $m_{1} \neq 0$,

$$
\widetilde{B}^{\left(m_{1}\right)}=\delta_{0, m_{1}} \hat{B}^{\left(m_{1}\right)}=0 .
$$

For a given positive integer value of $a$, the latter conditions enforce the vanishing of all coefficients

$$
\widetilde{B}_{\left(\left(k_{1} \ell_{1}\right) \ldots\left(k_{a} \ell a\right)\right)}^{\left(m_{1} \ldots m_{r}\right)} k_{b}, \ell_{c}, m_{s}= \pm 1, \pm 2, \ldots, \quad b, c=1, \ldots, a, \quad s=1, \ldots, r
$$

once the coefficients

$$
\widetilde{B}_{\left(\left(k_{1} \ell_{1}\right) \ldots\left(k_{a} \ell_{a}\right)\right)} k_{b}, \ell_{c}= \pm 1, \pm 2, \ldots, \quad b, c=1, \ldots, a
$$

vanish. Therefore, the claim will be proved if I can show that the assumption of the existence of a minimal positive integer $A$ with

$$
\sum_{k_{1}}^{\prime} \sum_{\ell_{1}}^{\prime} \ldots \sum_{k_{A}}^{\prime} \sum_{\ell_{A}}^{\prime}\left(\alpha_{i_{1}}^{k_{1}} \alpha_{i_{1}}^{\ell_{1}}\right) \ldots\left(\alpha_{i_{A}}^{k_{A}} \alpha_{i_{A} \ell_{A}}^{\ell_{1}} \widetilde{B}_{\left(\left(k_{1} \ell_{1}\right) \ldots\left(k_{A} \ell_{A}\right)\right)} \neq 0\right.
$$

leads to a contradiction.

Vanishing Poisson brackets of $\left(\mathscr{K}^{(1)}-\sum_{a=1}^{\infty} \beta_{a} \mathscr{C}^{a}\right)$ and the "renormalized" invariant charges $\mathscr{Z}_{i \underset{(2 p) \times}{(2)} \underbrace{}_{0} i 0}$ imply for all positive integer values of $p$ :

$$
\sum_{k_{1}}^{\prime} \sum_{\ell_{1}}^{\prime} \ldots \sum_{k_{A}}^{\prime} \sum_{\ell_{A}}^{\prime}\left(\alpha_{i_{1}}^{k_{1}} \alpha_{i_{1}}^{\ell_{1}}\right) \ldots\left(\alpha_{i_{A}}^{k_{A}} \alpha_{i_{A}}^{\ell}\right) \widetilde{B}_{\left(\left(k_{1} \ell_{1}\right) \ldots\left(k_{A} \ell_{A}\right)\right)}\left(\frac{1}{k_{1}^{2 p-1}}+\ldots+\frac{1}{\ell_{A}^{2 p-1}}\right)=0 .
$$

Hence $\widetilde{B}_{\left(\left(k_{1} \ell_{1}\right) \ldots\left(k_{A} \ell_{A}\right)\right)}=0$ except when the indices $k_{1}, l_{1}, \ldots, k_{A}, \ell_{A}$ can be grouped into pairs such that the values of the indices of each pair sum to zero.

Next, by taking into account the vanishing of the Poisson brackets of $\left(\mathscr{K}^{(1)}-\sum_{a=1}^{\infty} \beta_{a} \mathscr{C}^{a}\right)$ and the "renormalized" invariant charges $\mathscr{Z}_{j \underbrace{(2)}_{(2 p) \times} \ldots j}$ (no summation over $j$ !) $p=1,2,3, \ldots$, I want to argue that without loss of generality 
$\widetilde{B}_{\left(\left(k_{1} \ell_{1}\right) \ldots\left(k_{A} \ell_{A}\right)\right)}$ may be assumed to vanish unless $0=k_{1}+\ell_{1}=\ldots=k_{A}+\ell_{A}$. The vanishing of the latter type of Poisson brackets implies in particular that for any given set of values for the indices $k_{1}, \ell_{1}, \ldots, k_{A}, \ell_{A}$ which can be broken up into pairs such that the values of the indices of each pair sum to zero:

$$
\begin{aligned}
& \left(\frac{1}{k_{A}^{2 p-1}}+\frac{1}{\ell_{A}^{2 p-1}}\right) \cdot \sum_{k_{1}^{\prime}}^{\prime} \sum_{\ell_{1}^{\prime}}^{\prime} \ldots \sum_{k_{A-1}}^{\prime} \sum_{\ell_{A-1}^{\prime}}^{\sum_{A-1}^{\prime}}\left(\alpha_{i_{1} \neq j}^{k^{\prime}} \alpha_{i_{1} \neq j}^{\ell_{1}^{\prime}}\right) \ldots\left(\alpha_{i_{A-1} \neq j}^{k_{A}^{\prime}-1} \alpha_{i_{A-1} \ell^{\prime}-1}^{\prime}\right) \\
& \times \widetilde{B}_{\left(\left(k_{1}^{\prime}, \ell_{1}^{\prime}\right) \ldots\left(k_{A-1}^{\prime} \ell_{A-1}^{\prime}\right)\left(k_{A} \ell_{A}\right)\right)}=0, \\
& \left(\frac{1}{k_{A-1}^{2 p-1}}+\frac{1}{\ell_{A-1}^{2 p-1}}+\frac{1}{k_{A}^{2 p-1}}+\frac{1}{\ell_{A}^{2 p-1}}\right) \\
& \times \sum_{k_{1}}^{\prime} \sum_{\ell_{1}^{\prime}}^{\prime} \ldots \sum_{k_{A-2}^{\prime}}^{\prime} \sum_{\ell_{A-2}^{\prime}}^{\prime}\left(\alpha_{i_{1} \neq j}^{k_{1}^{\prime}} \alpha_{i_{1} \neq j}^{\ell_{1}^{\prime}}\right) \ldots\left(\alpha_{i_{A-2} \neq j}^{k_{A}^{\prime}-2} \alpha_{i_{A-2} \neq j}^{\ell_{A-2}^{\prime}}\right)
\end{aligned}
$$

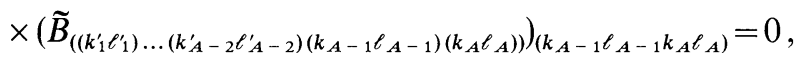

$$
\begin{aligned}
& \left(\frac{1}{k_{2}^{2 p-1}}+\frac{1}{\ell_{2}^{2 p-1}}+\ldots+\frac{1}{k_{A}^{2 p-1}}+\frac{1}{\ell_{A}^{2 p-1}}\right) \sum_{\hat{k}_{1}^{\prime}}^{\prime} \sum_{\ell_{1}^{\prime}}^{\prime}\left(\alpha_{i_{1} \neq j}^{k_{1}^{\prime}} \alpha_{i_{1} \neq j}^{\ell_{1}^{\prime}}\right) \\
& \times\left(\widetilde{B}_{\left(\left(k_{1}^{\prime} \ell^{\prime}\right)\left(k_{2} \ell_{2}\right) \ldots\left(k_{A} \ell_{A}\right)\right)}\right)_{\left(k_{2} \ell_{2} \ldots k_{A} \ell_{A}\right)}=0, \quad p=1,2,3, \ldots
\end{aligned}
$$

The consequences of the first of these equations can be recognized most easily when the equation is multiplied by $\alpha_{i_{A} \neq j}^{k_{A}} \alpha_{i_{A} \neq j}^{\ell_{A}}$ and by $\alpha_{j}^{k_{A}} \alpha_{j}^{\ell_{A}}$ and finally summed over in the indices $k_{A}$ and $l_{A}$

a)

$$
\begin{aligned}
& \sum_{k_{1}}^{\prime} \sum_{\ell_{1}}^{\prime} \ldots \sum_{k_{A}}^{\prime} \sum_{\ell_{A}}^{\prime}\left(\alpha_{i_{1} \neq j}^{k_{1}} \alpha_{i_{1} \neq j}^{\ell_{1}}\right) \ldots\left(\alpha_{i_{A} \neq j}^{k_{A}} \alpha_{i_{A} \neq j}^{\ell_{A}}\right) \widetilde{B}_{\left(\left(k_{1} \ell_{1}\right) \ldots\left(k_{A} \ell_{A}\right)\right)} \\
& =\sum_{k_{1}}^{\prime} \sum_{\ell_{1}}^{\prime} \ldots \sum_{k_{A-1}}^{\prime} \sum_{\ell_{A-1}^{\prime}}^{\prime} \sum_{k_{A}}^{\prime}\left(\alpha_{i_{1} \neq j}^{k_{1}} \alpha_{i_{1} \neq j}^{\ell_{1}}\right) \ldots\left(\alpha_{i_{A-1} \neq j}^{k_{A-1}} \alpha_{i_{A-1} \neq j}^{\ell_{A-1}}\right) \\
& \times\left(\alpha_{i_{A} \neq j}^{k_{A} \neq \alpha_{i_{A} \neq j}} \alpha^{-k_{A}} \tilde{B}_{\left(\left(k_{1} \ell_{1}\right) \ldots\left(k_{A} \ell_{A}\right)\right) / k_{A}+\ell_{A}=0}\right.
\end{aligned}
$$

and

b)

$$
\begin{aligned}
& \sum_{k_{1}}^{\prime} \sum_{\ell_{1}}^{\prime} \ldots \sum_{k_{A}}^{\prime} \sum_{\ell_{A}}^{\prime}\left(\alpha_{i_{1} \neq j}^{k_{1}} \alpha_{i_{1} \neq j}^{\ell_{1}}\right) \ldots\left(\alpha_{i_{A-1} \neq j}^{k_{A-1}} \alpha_{i_{A}-1 \neq j}^{\ell_{A-1}}\right) \\
& \times\left(\alpha_{j}^{k_{A}} \alpha_{j}^{\ell_{A}}\right) \widetilde{B}_{\left(\left(k_{1} \ell_{1}\right) \ldots\left(k_{A} \ell_{A}\right)\right)} \\
& =\sum_{k_{1}}^{\prime} \sum_{\ell_{1}}^{\prime} \ldots \sum_{k_{A-1}}^{\prime} \sum_{\ell_{A-1}^{\prime}}^{\prime} \sum_{k_{A}}^{\prime}\left(\alpha_{i_{1} \neq j}^{k_{1}} \alpha_{i \neq j}^{\ell_{1}}\right) \ldots\left(\alpha_{i_{A-1} \neq j}^{k_{A-1}} \alpha_{i_{A-1} \neq j}^{\ell_{A-1}}\right) \\
& \times\left(\alpha_{j}^{k_{A}} \alpha_{j}^{\left.-k_{A}\right)} \widetilde{B}_{\left(\left(k_{1} \ell_{1}\right) \ldots\left(k_{A} \ell_{A}\right)\right) / k_{A}+\ell_{A}=0} .\right.
\end{aligned}
$$

In words: Nonvanishing contributions to

$$
\sum_{k_{1}}^{\prime} \sum_{\ell_{1}}^{\prime} \ldots \sum_{k_{A}}^{\prime} \sum_{\ell_{A}}^{\prime}\left(\alpha_{i_{1}}^{k_{1}} \alpha_{i_{1}}^{\ell_{1}}\right) \ldots\left(\alpha_{i_{A}}^{k_{A}} \alpha_{i_{A}}^{\ell_{A}}\right) \widetilde{B}_{\left(\left(k_{1} \ell_{1}\right) \ldots\left(k_{A} \ell_{A}\right)\right)}
$$

from symmetry types in the coefficients $\widetilde{B}_{\left(\left(k_{1} \ell_{1}\right) \ldots\left(k_{A} \ell_{A}\right)\right)}$ which correspond to Young tableaux with a) less than $(d-1)$ rows, and $b$ ) with $(d-1)$ rows and two boxes in the bottom row arise only from those values of the indices for which $k_{A}+\ell_{A}=0$. 
Similarly, the second of the above equations can be cast into the form

$$
\begin{aligned}
& \sum_{k_{1}}^{\prime} \sum_{\ell_{1}}^{\prime} \ldots \sum_{k_{A}}^{\prime} \sum_{\ell_{A}}^{\prime}\left(\alpha_{i_{1} \neq j}^{k_{1}} \alpha_{i_{1} \neq j}^{\ell_{1}}\right) \ldots\left(\alpha_{i_{A-2} \neq j}^{k_{A-2}} \alpha_{i_{A-2} \ell_{A-2}}^{\ell_{A}}\right)\left(\alpha_{j}^{k_{A-1}} \alpha_{j}^{\ell_{A}-1} \alpha_{j}^{k_{A}} \alpha_{j}^{\ell_{A} A}\right) \\
& \times \widetilde{B}_{\left(\left(k_{1} \ell_{1}\right) \ldots\left(k_{A} \ell_{A}\right)\right)}=\sum_{k_{1}}^{\prime} \sum_{\ell_{1}}^{\prime} \ldots \sum_{k_{A-2}}^{\prime} \sum_{\ell=2}^{\prime} \sum_{k_{A-1}}^{\prime} \sum_{\ell_{A-1}}^{\prime} \sum_{k_{A}}^{\prime} \sum_{\ell_{A}}^{\prime}\left(\alpha_{i_{1} \neq j}^{k_{1}} \alpha_{i_{1} \neq j}^{\ell_{1}}\right) \ldots \\
& \ldots\left(\alpha_{i_{A-2} \neq j}^{k_{A-2}} \alpha_{i_{A-2} \ell_{A-2}}\right)\left(\alpha_{j}^{k_{A-1}} \alpha_{j}^{\ell_{A}-1} \alpha_{j}^{k_{A}} \alpha_{j}^{\ell_{A}}\right) \\
& \times\left(\widetilde{B}_{\left(\left(k_{1} \ell_{1}\right) \ldots\left(k_{A} \ell_{A}\right)\right)}\right)_{\left(k_{A-1} \ell_{A-1} k_{A} \ell_{A}\right)}\left[\delta\left(k_{A-1}+\ell_{A-1}\right) \delta\left(k_{A}+\ell_{A}\right)\right. \\
& +\delta\left(k_{A-1}+k_{A}\right) \delta\left(\ell_{A-1}+\ell_{A}\right)+\delta\left(k_{A-1}+\ell_{A}\right) \delta\left(\ell_{A-1}+k_{A}\right) \\
& -\delta\left(k_{A-1}+\ell_{A-1}\right) \delta\left(k_{A-1}+k_{A}\right) \delta\left(k_{A-1}-\ell_{A}\right) \\
& -\delta\left(k_{A-1}+k_{A}\right) \delta\left(k_{A-1}+\ell_{A}\right) \delta\left(k_{A-1}-\ell_{A-1}\right) \\
& \left.-\delta\left(k_{A-1}+\ell_{A}\right) \delta\left(k_{A-1}+\ell_{A-1}\right) \delta\left(k_{A-1}-k_{A}\right)\right] \text {, }
\end{aligned}
$$

where

$$
\delta(k)=\delta_{0, k} .
$$

In words: Non-vanishing contributions to

$$
\sum_{k_{1}}^{\prime} \sum_{\ell_{1}}^{\prime} \ldots \sum_{k_{A}}^{\prime} \sum_{\ell_{A}}^{\prime}\left(\alpha_{i_{1}}^{k_{1}} \alpha_{i_{1}}^{\ell_{1}}\right) \ldots\left(\alpha_{i_{A}}^{k_{A}} \alpha_{i_{A}{ }_{A}}^{\ell}\right) \widetilde{B}_{\left(\left(k_{1} \ell_{1}\right) \ldots\left(k_{A} \ell_{A}\right)\right)}
$$

from symmetry types of the coefficients $\widetilde{B}_{\left(\left(k_{1} \ell_{1}\right) \ldots\left(k_{A} \ell_{A}\right)\right)}$ which correspond to Young tableaux with $(d-1)$ rows and four boxes in the bottom row arise only from those values of the indices for which the set $k_{A-1}, \ell_{A-1}, k_{A}, \ell_{A}$ can be split into pairs such that the values of the indices of each pair sum to zero.

Now, observe that the coefficients $\widetilde{B} \ldots$ (of the specified symmetry type) enter the above sum in the symmetrized form $(\widetilde{B} \ldots)_{\left(k_{A-1} \ell_{A-1} k_{A} \ell_{A}\right)}$ with a correspondingly symmetric support only. However, such coefficients can be generated by symmetrization in the indices $k_{A-1}, \ell_{A-1}, k_{A}, \ell_{A}$ of coefficients which vanish unless $0=k_{A-1}+\ell_{A-1}=k_{A}+\ell_{A}$. Thus, also for the specified symmetry type, without loss of generality $\widetilde{B}_{\left(\left(k_{1} \ell_{1}\right) \ldots\left(k_{A} \ell_{A}\right)\right)}$ may be assumed to vanish unless $k_{A}+\ell_{A}=0$. Using similar arguments also for the remaining symmetry types, I conclude that indeed $\widetilde{B}_{\left(\left(k_{1} \ell_{1}\right) \ldots\left(k_{A} \ell_{A}\right)\right)}$ may be assumed to vanish unless $k_{A}+\ell_{A}=0$.

From the symmetry of the coefficients $\widetilde{B}_{\left(\left(k_{1} \ell_{1}\right) \ldots\left(k_{A} \ell_{A}\right)\right)}$ under the interchange of $k_{b}$ and $\ell_{b}, b=1, \ldots, A$ and under the interchange of any two pairs $\left(k_{b}, \ell_{b}\right)$ and $\left(k_{c}, \ell_{c}\right)$, $b, c=1, \ldots, A$, it follows that $\widetilde{B}_{\left(\left(k_{1} \ell_{1}\right) \ldots\left(k_{A} \ell_{A}\right)\right)}$ vanishes unless the indices take values which satisfy $0=k_{1}+\ell_{1}=\ldots=k_{A}+\ell_{A}$ :

$$
\widetilde{B}_{\left(\left(k_{1} \ell_{1}\right) \ldots\left(k_{A} \ell_{A}\right)\right)}=\delta\left(k_{1}+\ell_{1}\right) \ldots \delta\left(k_{A}+\ell_{A}\right) \cdot \hat{B}\left(k_{1}^{2}, \ldots, k_{A}^{2}\right) / 2^{A},
$$

where for any permutation $\pi$ from the symmetric group $\mathfrak{\Xi}^{A}$ :

$$
\hat{B}\left(k_{\pi(1)}^{2}, \ldots, k_{\pi(A)}^{2}\right)=\hat{B}\left(k_{1}^{2}, \ldots, k_{A}^{2}\right) \text {. }
$$

Hence,

$$
\begin{aligned}
& \sum_{k_{1}}^{\prime} \sum_{\ell_{1}}^{\prime} \ldots \sum_{k_{A}}^{\prime} \sum_{\ell_{A}}^{\prime}\left(\alpha_{i_{1}}^{k_{1}} \alpha_{i_{1}}^{\ell_{1}}\right) \ldots\left(\alpha_{i_{A}}^{k} \alpha_{i_{A}}^{\ell_{A}}\right) \widetilde{B}_{\left(\left(k_{1} \ell_{1}\right) \ldots\left(k_{A} \ell_{A}\right)\right)} \\
& \quad=\sum_{k_{1}=1}^{\infty} \ldots \sum_{k_{1}=1}^{\infty}\left(\alpha_{i_{1}}^{k_{1}} \alpha_{i_{1}}^{-k_{1}}\right) \ldots\left(\alpha_{i_{A}}^{k_{A}} \alpha_{i_{A}}^{-k_{A}}\right) \hat{B}\left(k_{1}^{2}, \ldots, k_{A}^{2}\right) .
\end{aligned}
$$


Now, in a final step, I want to argue that $\hat{B}\left(k_{1}^{2}, \ldots, k_{A}^{2}\right)$ must be constant, in fact, that it must vanish. To this end consider the Poisson bracket of $\left(\mathscr{K}^{(1)}-\sum_{a=1}^{\infty} \beta_{a} \mathscr{C}^{a}\right)$ with the "renormalized" invariant charge $\mathscr{Z}_{0 i j i}^{(2)}$ :

$$
\mathscr{Z}_{0 i j i i}^{(2)}=-(2 \pi)^{2} \sum_{m}^{\prime} \sum_{n}^{\prime} \frac{\alpha_{i}^{m} \alpha_{i}^{n}}{(i m)(i n)} \alpha_{j}^{-(m+n)}+O\left(\alpha_{i}^{k} \alpha_{j}^{\ell} \alpha_{i}^{m} \alpha_{0}^{n}\right),
$$

where $O\left(\alpha_{i}^{k} \alpha_{j}^{\ell} \alpha_{i}^{m} \alpha_{0}^{n}\right)$ denotes the contribution of first degree in the variables

$$
\alpha_{i}^{k} \alpha_{j}^{\ell} \alpha_{i}^{m} \alpha_{0}^{n}, \quad k, \ell, m, n= \pm 1, \pm 2, \ldots .
$$

The Poisson bracket in question must vanish. Hence, in particular

$$
\begin{aligned}
\sum_{k_{1}=1}^{\infty} \ldots \sum_{k_{A-1}=1}^{\infty} \sum_{\substack{m \\
m+n}}^{\prime} \sum_{n=0}^{\prime}\left(\alpha_{i_{1}}^{k_{1}} \alpha_{i_{1}}^{-k_{1}}\right) \ldots\left(\alpha_{i_{A-1}}^{k_{A}-1} \alpha_{i_{A}-1}^{-k_{A-1}}\right) \frac{\left(\alpha_{i_{A}}^{m} \alpha_{i_{A}}^{n}\right)}{(i m)(i n)} \alpha_{j}^{-(m+n)} \\
\quad \times\left[(m+n) \hat{B}\left(k_{1}^{2}, \ldots, k_{A-1}^{2},(m+n)^{2}\right)-m \hat{B}\left(k_{1}^{2}, \ldots, k_{A-1}^{2}, m^{2}\right)\right. \\
\left.\quad-n \hat{B}\left(k_{1}^{2}, \ldots, k_{A-1}^{2}, n^{2}\right)\right]=0 .
\end{aligned}
$$

This in turn implies the identity $\left(k_{b}^{2} \neq 0\right.$ for all $\left.b=1, \ldots, A-1\right)$

$$
(m+n) \hat{B}\left(k_{1}^{2}, \ldots, k_{A-1}^{2},(m+n)^{2}\right)=m \hat{B}\left(k_{1}^{2}, \ldots, k_{A-1}^{2} m^{2}\right)+n \hat{B}\left(k_{1}^{2}, \ldots, k_{A-1}^{2}, n^{2}\right) .
$$

The justifivation for the latter conclusion derives from the observation that the only solutions of the equation $(d \geqq 3$ !)

$$
\begin{aligned}
& \left(\alpha_{i_{1}}^{k_{1}} \alpha_{i_{1}}^{-k_{1}}\right) \ldots\left(\alpha_{i_{A-1}}^{k_{A-1}} \alpha_{i_{A}-1}^{-k_{A-1}}\right)\left(\alpha_{i_{A}}^{m} \alpha_{i_{A}}^{n}\right) \alpha_{j}^{-(m+n)} \\
& \quad=\left(\alpha_{i_{1}}^{k_{1}^{\prime}} \alpha_{i_{1}}^{-k_{1}^{\prime}}\right) \ldots\left(\alpha_{i_{A}-1}^{k_{A}-1} \alpha_{i_{A}-1}^{-k_{A}^{\prime}-1}\right)\left(\alpha_{i_{A}}^{m^{\prime}} \alpha_{i_{A}}^{n^{\prime}}\right) \alpha_{j}^{-\left(m^{\prime}+n^{\prime}\right)},
\end{aligned}
$$

$k_{b}, k_{b^{\prime}}^{\prime}, b, b^{\prime}=1, \ldots, A-1$ all positive integers, $m, n\left(m^{\prime}, n^{\prime}\right)$ non-zero integers which do not sum to zero, and the Fourier coefficients $\alpha_{i}^{\ell}, \ell= \pm 1, \pm 2, \ldots, i=1,2, \ldots, d-1$ treated as independent variables, are:

$$
k_{b}^{\prime}=k_{\pi(b)}, \quad b=1,2, \ldots, A-1, m^{\prime}=m, n^{\prime}=n
$$

and

$$
k_{b}^{\prime}=k_{\pi(b)}, \quad b=1,2, \ldots, A-1, m^{\prime}=n, n^{\prime}=m
$$

for some permutation $\pi$ from the symmetric group $\mathfrak{\subseteq}^{A-1}$. It follows that $\hat{B}\left(k_{1}^{2}, k_{2}^{2}, \ldots, k_{A-1}^{2}, k_{A}^{2}\right)$ does not depend on the value of $k_{A}^{2}$. But by symmetry this implies that $\hat{B}\left(k_{1}^{2}, \ldots, k_{A}^{2}\right)$ does not depend on the value of any $k_{b}^{2}, b=1, \ldots, A$. In other words $\hat{B}\left(k_{1}^{2}, \ldots, k_{A}^{2}\right)$ must be a constant equal to $\hat{B}(1, \ldots, 1)$. Now, due to the convention concerning the choice of the coefficients $\beta_{a}, a=1,2, \ldots$,

$$
0=\widetilde{B}_{((1,-1) \ldots(1,-1))}=\hat{B}(1,1, \ldots, 1) .
$$

Hence:

$$
\hat{B}\left(k_{1}^{2}, \ldots, k_{A}^{2}\right) \equiv 0,
$$

and finally in contradiction to the defining property of $A$ :

$$
\sum_{k_{1}}^{\prime} \sum_{\ell_{1}}^{\prime} \ldots \sum_{k_{A}}^{\prime} \sum_{\ell_{A}}^{\prime}\left(\alpha_{i_{1}}^{k_{1}} \alpha_{i_{1}}^{\ell_{1}}\right) \ldots\left(\alpha_{i_{A}}^{k_{A}} \alpha_{i_{A}}^{\ell_{A}}\right) \widetilde{B}_{\left(\left(k_{1} \ell_{1}\right) \ldots\left(k_{A} \ell_{A}\right)\right)}=0 .
$$


This completes the proof that any Casimir element of the scalar type contained in the formal closure of $\mathfrak{h}_{(m, 0, \ldots, 0)}$ is a function of the special Casimir element $\mathscr{C}$.

\section{Conclusions}

According to the above analysis, each one of the two algebras of invariant charges $\mathfrak{h}_{\mathscr{P}}^{ \pm}$contains in its formal closure exactly one independent Casimir element

$$
\mathscr{C}^{+}=\oint_{\tau=\text { const }} d \sigma\left[\left(u^{+\mu}(\tau, \sigma) u_{\mu}^{+}(\tau, \sigma)\right)^{2}\right]^{1 / 4}
$$

and

$$
\mathscr{C}^{-}=\oint_{\tau=\text { const }} d \sigma\left[\left(u^{-\mu}(\tau, \sigma) u_{\mu}^{-}(\tau, \sigma)\right)^{2}\right]^{1 / 4}
$$

respectively. The representations of the algebras $\mathfrak{h}_{\mathscr{P}}^{+}$and $\mathfrak{h}_{\mathscr{P}}^{-}$relevant for the Nambu-Goto string theory are characterized by the conditions $\mathscr{C}^{+}=0$ and $\mathscr{C}^{-}=0$ corresponding to the invariant measures $\delta\left(\mathscr{C}^{+}\right)$and $\delta\left(\mathscr{C}^{-}\right)$. It is for this representation $\left(\mathscr{C}^{ \pm}=0\right.$ implies $\left.\sum_{\substack{k \\ k+\ell=p}} \sum_{\ell} \alpha^{ \pm \mu k} \alpha_{\mu}^{ \pm \ell}=0\right)$ that the limiting elements

$$
\begin{aligned}
\mathscr{I}_{a}^{ \pm}= & +\sum_{b=1}^{a}(-1)^{b}\left(\begin{array}{l}
a \\
b
\end{array}\right) \sum_{r=0}^{\infty} \frac{(-1)^{r}}{\mathrm{~m}^{r}} \frac{\Gamma(2 b-1+r)}{\Gamma(2 b-1) \cdot r !} \\
& \times \sum_{k_{1}} \sum_{\ell_{1}} \ldots \sum_{k_{b}} \sum_{\ell_{b}} \sum_{m_{1}}^{\prime} \ldots \sum_{m_{r}}^{\prime}\left(\alpha^{ \pm \mu_{1} k_{1}} \alpha_{\mu_{1}}^{ \pm \ell_{1}}\right) \ldots\left(\alpha^{ \pm \mu_{b} k_{b}} \alpha_{\mu_{b}}^{ \pm \ell_{b}}\right) \\
& \times\left(\mathscr{P}^{\lambda_{1}} \alpha_{\lambda_{1}}^{ \pm m_{1}}\right) \ldots\left(\mathscr{P}^{\lambda_{r}} \alpha_{\lambda_{r}}^{ \pm m_{r}}\right) \delta\left(k_{1}+\ldots+\ell_{b}+m_{1}+\ldots+m_{r}\right)
\end{aligned}
$$

become multiples of 1 . Actually they become equal to 1. Due to the Poisson brackets of $\mathscr{I}_{a}^{ \pm}$with the invariant charges $\mathscr{Z}^{ \pm} \ldots$ :

$$
\begin{aligned}
\left\{\mathscr{I}_{a}^{ \pm}, \mathscr{Z}^{ \pm} \ldots\right\}= & \sum_{b=}^{a}(-1)^{b-1}\left(\begin{array}{l}
a \\
b
\end{array}\right) \sum_{r=0}^{\infty} \frac{(-1)^{r}}{m^{r+1}} \frac{\Gamma(2 b+r)}{\Gamma(2 b-1) r !} \\
& \times \sum_{k_{1}} \sum_{\ell_{1}} \ldots \sum_{k_{b}} \sum_{\ell_{b}} \sum_{m_{1}}^{\prime} \ldots \sum_{m_{r+1}}^{\prime}\left(\alpha^{ \pm \mu_{1} k_{1}} \alpha_{\mu_{1}}^{ \pm \ell_{1}}\right) \ldots \\
& \ldots .\left(\alpha^{ \pm \mu_{b} k_{b}} \alpha_{\mu_{b}}^{ \pm \ell_{b}}\right)\left(\mathscr{P}^{\lambda_{1}} \alpha_{\lambda_{1}}^{ \pm m_{1}}\right) \ldots\left(\mathscr{P}^{\lambda_{r}} \alpha_{\lambda_{r}}^{ \pm m_{r}}\right) \\
& \times \mathscr{P}^{\lambda_{r+1}}\left\{\alpha_{\lambda_{r+1}}^{ \pm m_{r+1}}, \mathscr{Z}^{ \pm} \ldots\right\} \\
& \times \delta\left(k_{1}+\ell_{1}+\ldots+k_{b}+\ell_{b}+m_{1}+\ldots+m_{r+1}\right)
\end{aligned}
$$

and, in particular, due to the vanishing of the Poisson brackets of the limiting elements $\mathscr{I}_{a}^{ \pm}$among each other, consistency is guaranteed. For the algebras of invariant charges $\mathfrak{h} \frac{ \pm}{\mathscr{P}}$, the reparametrization invariant operator conditions $\mathscr{I}_{a}^{ \pm}-1=0$ replace the non-invariant conditions $\sum_{\substack{k \\ k+\ell=p}} \sum_{\ell} \alpha^{ \pm \mu k} \alpha_{\mu}^{ \pm \ell}=0$ (which, of course, cannot be expressed in terms of invariant charges).

Thus, at least for the case $\mathscr{P}^{2}=\mathrm{m}^{2}>0$, I have confirmed the suggestion of [1] that the loop wave equations $u^{ \pm \mu}(\tau, \sigma) u_{\mu}^{ \pm}(\tau, \sigma)=0$ of the Nambu-Goto theory can and should be interpreted as an infinite collection of representation conditions related to requirements imposed on the Casimir elements of $\mathfrak{h} \frac{ \pm}{\mathscr{P}}$. 
Acknowledgements. I would like to thank the staff of the Physics Department of the University of California at Berkeley for the kind hospitality extended to me during the fall of 1986. Financial support by Stiftung Volkswagenwerk and Deutsche Forschungsgemeinschaft is gratefully acknowledged.

\section{References}

1. Pohlmeyer, K.: A group-theoretical approach to the quantization of the free relativistic closed string. Phys. Lett. 119B, 100 (1982)

2. Pohlmeyer, K., Rehren, K.-H.: The invariant charges of the Nambu-Goto theory: their geometric origin and their completeness. Commun. Math. Phys. 114, 177-186

3. Pohlmeyer, K.: The invariant charges of the Nambu-Goto theory in WKB-approximation: renormalization. Commun. Math. Phys. 105, 629 (1986)

4. Pohlmeyer, K., Rehren, K.H.: Algebraic properties of the invariant charges of the Nambu-Goto theory. Commun. Math. Phys. 105, 593 (1986)

5. Pohlmeyer, K., Rehren, K.-H.: The algebra formed by the invariant charges of the NambuGoto theory: identification of a maximal abelian subalgebra. Commun. Math. Phys. 114, 55-68

6. Dixmier, J.: Enveloping algebras. Mathematical Library Vol. 14. Amsterdam: North-Holland 1977

Communicated by K. Osterwalder

Received April 21, 1987 